\title{
Universiteit
}

Leiden

The Netherlands

\section{Emission of polarization-entangled microwave photons from a pair of quantum dots}

Emary, C.; Trauzettel, B.; Beenakker, C.W.J.

\section{Citation}

Emary, C., Trauzettel, B., \& Beenakker, C. W. J. (2005). Emission of polarization-entangled microwave photons from a pair of quantum dots. Physical Review Letters, 95(12), 127401. doi:10.1103/PhysRevLett.95.127401

Version: $\quad$ Not Applicable (or Unknown)

License: $\quad$ Leiden University Non-exclusive license

Downloaded from: https://hdl.handle.net/1887/64334

Note: To cite this publication please use the final published version (if applicable). 


\title{
Emission of Polarization-Entangled Microwave Photons from a Pair of Quantum Dots
}

\author{
C. Emary, B. Trauzettel, and C. W. J. Beenakker \\ Instituut-Lorentz, Universiteit Leiden, P.O. Box 9506, 2300 RA Leiden, The Netherlands
}

(Received 23 February 2005; published 12 September 2005)

\begin{abstract}
We describe a mechanism for the production of polarization-entangled microwaves using intraband transitions in a pair of quantum dots. This proposal relies neither on spin-orbit coupling nor on control over electron-electron interactions. The quantum correlation of microwave polarizations is obtained from orbital degrees of freedom in an external magnetic field. We calculate the concurrence of emitted microwave photon pairs and show that a maximally entangled Bell pair is obtained in the limit of weak interdot coupling.
\end{abstract}

DOI: 10.1103/PhysRevLett.95.127401

PACS numbers: 78.67.Hc, 42.65.Lm, 78.70.Gq

Entangled photons at optical frequencies are routinely produced by nonlinear optical effects in macroscopic crystals [1]. The use of semiconductor nanostructures to produce these states promises both a greater frequency range and a closer integration with quantum electronics.

One way to produce entangled photons is to start with entangled electrons and then transfer this entanglement [2]. However, one can also start with nonentangled particles, and most work in this area has focused on the decay of biexcitonic states in quantum dots [3-6]. In the original proposal [3], a biexciton is formed in a single dot by electrical pumping, and then subsequently decays by emitting a pair of photons via one of two cascades. The polarizations of these two photons are linked to the cascade by which the biexciton decays, and thus, if these cascades proceed coherently, one produces polarization-entangled photons. All these proposals [2-6] involve interband transitions between valence and conduction bands of a quantum dot, so that the output photons have frequencies in the visible range.

In this Letter, we propose the use of intraband transitions of conduction band electrons to generate entangled microwave photons. Our proposal, illustrated in Fig. 1, can be seen as the real-space analogue of the biexciton decay cascade in energy of Ref. [3]. The microwaves originate from spontaneous downward transitions between singleparticle levels in a quantum dot. That these transitions couple to microwaves has been demonstrated in photonassisted tunneling experiments [7].

Our entanglement scheme requires four quantum dots as shown in Fig. 1: two dots $(L, R)$ to provide unique initial and final states for the electron, and two more dots $(A, B)$ to provide the two decay paths. For real symmetric tunnel couplings, an electron tunnels through the single level in $\operatorname{dot} L$ into an equal superposition $2^{-1 / 2}\left(\left|A^{*}\right\rangle+\left|B^{*}\right\rangle\right)$ of upper levels in dots $A$ and $B$. It decays to the ground state with the emission of two photons. The dots are configured such that the same process gives rise to two left circularly polarized $\left(\mathrm{CP}^{+}\right)$photons in $\operatorname{dot} A$ and two right circularly polarized $\left(\mathrm{CP}^{-}\right)$photons in $\operatorname{dot} B$. (We will describe later how this can be done.) The resulting state
$|\Psi\rangle=2^{-1 / 2}\left(\left|A_{G}\right\rangle|++\rangle+\left|B_{G}\right\rangle|--\rangle\right)$ encodes the state of the quantum dot onto pairs of photons with left or right circular polarization.

To disentangle the photons from the electrons, we couple dots $A$ and $B$ symmetrically to a fourth dot $R$, drained by an electron reservoir. This construction effectively projects the electronic state in dots $A$ and $B$ onto the even combination $\left|\Phi_{+}\right\rangle=2^{-1 / 2}\left(\left|A_{G}\right\rangle+\left|B_{G}\right\rangle\right)$, since destructive interference prevents the odd combination $\left|\Phi_{-}\right\rangle=2^{-1 / 2}\left(\left|A_{G}\right\rangle-\left|B_{G}\right\rangle\right)$ from tunneling coherently into dot $R$. Defining also the even and odd combinations $\left|\Psi_{ \pm}\right\rangle=2^{-1 / 2}(|++\rangle \pm|--\rangle)$ of the photon states, we may write $|\Psi\rangle=2^{-1 / 2}\left(\left|\Phi_{+}\right\rangle\left|\Psi_{+}\right\rangle+\left|\Phi_{-}\right\rangle\left|\Psi_{-}\right\rangle\right)$. The projection of $|\Psi\rangle$ onto $\left|\Phi_{+}\right\rangle$thus produces the required entangled photon pair $\left|\Psi_{+}\right\rangle$.

While not strictly necessary for the entangling mechanism, it is helpful to assume that the quantum dots are inserted into a cylindrical microwave resonator able to support both polarizations at the resonant frequency $\Omega$. Recent results for double quantum dots and superconducting transmission line resonators have shown the possibility of extremely large dot-microwave couplings $[8,9]$. The cavity ensures that microwave, and not phonon, transitions dominate, and also serves to counteract any slight nonidealities in the quantum dot emission frequencies that might otherwise render the two decay paths distinguish-

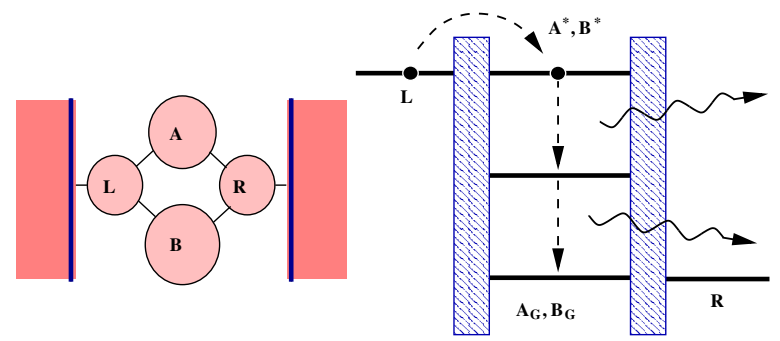

FIG. 1 (color online). Schematic of the microwave entangler. Left panel: The four-dot arrangement between two electron reservoirs. Right panel: Positions of dot levels, with arrows indicating the transitions discussed in the text. 
able. But most importantly, the cavity allows operation of the device without postselection.

In the absence of the cavity, it is necessary to detect whether the electron escapes into the right reservoir after having produced a photon pair, in order to effectuate the projection onto $\left|\Phi_{+}\right\rangle$. If the electron remains trapped in the state $\left|\Phi_{-}\right\rangle$, the photon pair should be discarded. This postselection discards one out of two attempts - even under ideal conditions. As we will show in what follows, it is possible to entirely avoid the postselection by using a microwave resonator to evolve $\left|\Phi_{-}\right\rangle\left|\Psi_{-}\right\rangle$into $\left|\Phi_{+}\right\rangle\left|\Psi_{+}\right\rangle$. The electron will then always escape into the reservoir, and the required state $\left|\Psi_{+}\right\rangle$is produced at each and every attempt. The resultant entanglement could be detected via the violation of a Bell inequality. This is a routine experiment for visible light; the analogous experiment at microwave frequencies is an experimental challenge, with some recent progress [10].

After these qualitative considerations, we now turn to a quantitative description. The two emitting quantum dots, $A$ and $B$, are assumed to have cylindrically symmetric, parabolic confining potentials. A perpendicular field $B_{0}$ is applied to control the microwave polarizations. The dots are set in a small area such that the charging energy is sufficient to prevent more than one electron being in the four-dot system at any time. Dots $A$ and $B$ are in tunneling contact with the single levels in dots $L$ and $R$. The state $E_{L}$ of dot $L$ is aligned with the levels $E_{1}$ in dots $A$ and $B$, and the single level $E_{R}$ in dot $R$ is aligned with the ground states at $E_{3}$ (cf. Fig. 2). The chemical potentials of the electron reservoirs are adjusted such that on the left $\mu_{L} \gg$ $E_{L}$ and on the right $\mu_{R} \ll E_{R}$.

Since we never occupy the dots with more than one electron at a time, a spinless single-particle picture is appropriate. The Hamiltonian of quantum dot $Y=A, B$ is

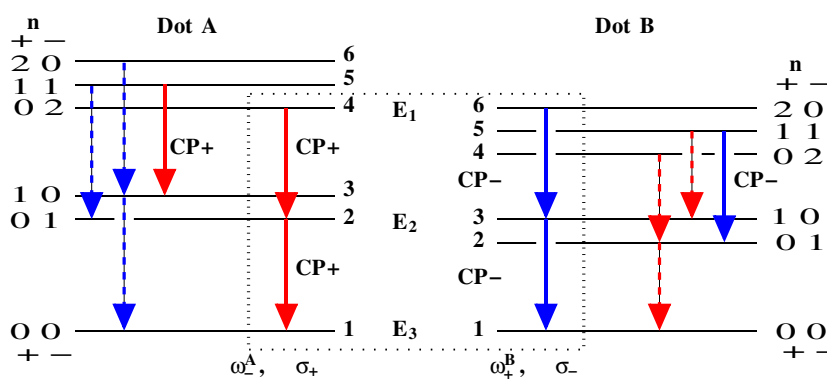

FIG. 2 (color online). Fock-Darwin spectrum of dots $A$ and $B$ : the six lowest levels with their quantum numbers $n_{ \pm}$are shown for each dot. The dot sizes are tuned such that $\omega_{-}^{A}=\omega_{+}^{B}=\Omega$. Selection rule allowed transitions are shown, with solid (dashed) arrows denoting on (off) resonant transitions. The circular polarization, $\mathrm{CP}^{+}$or $\mathrm{CP}^{-}$, of the emitted microwaves is also shown. In the entangler, electrons are injected into the two levels at $E_{1}$, and decay via the transitions shown in the dotted rectangle. In $\operatorname{dot} A$, this produces a cascade of two $\mathrm{CP}^{+}$photons, whereas in $\operatorname{dot} B$ two $\mathrm{CP}^{-}$photons are produced.

$$
H_{Y}=\omega_{+}^{Y} a_{+}^{Y \dagger} a_{+}^{Y}+\omega_{-}^{Y} a_{-}^{Y \dagger} a_{-}^{Y}=\sum_{i} \varepsilon_{i}^{Y}\left|Y_{i}\right\rangle\left\langle Y_{i}\right|
$$

The excitation energies are $\omega_{ \pm}^{Y}=\sqrt{\omega_{0}^{Y 2}+\omega_{c}^{2} / 4} \pm \omega_{c} / 2$, with $\omega_{c}=e B_{0} / m$ the cyclotron frequency and $\omega_{0}^{Y}$ the confinement energy of $\operatorname{dot} Y$ (we set $\hbar=1$ ). This so-called Fock-Darwin spectrum [11] is thus determined by the two quantum numbers $n_{ \pm}^{Y}$ [12]. Electric dipole transitions between dot levels with photon emission along the symmetry axis satisfy the selection rule $\left|\Delta n_{ \pm}\right|=1$. Transitions in which $n_{-}$decreases emit $\mathrm{CP}^{+}$photons, and transitions in which $n_{+}$decreases emit $\mathrm{CP}^{-}$photons.

Figure 2 shows the six lowest levels $\left|Y_{i}\right\rangle$ in dots $Y=A, B$, labeled $i=1$ to 6 from the bottom up. We take $B_{0}$ small enough that there are no level crossings in the spectrum. Since $\omega_{ \pm}$depends on the confinement energy $\omega_{0}$, the spectra of the dots can be tuned by electrostatically changing their sizes. We set $\omega_{-}$of $\operatorname{dot} A$ equal to $\omega_{+}$of dot $B$ by choosing the ratio of the linear sizes to be $l_{A} / l_{B} \approx 1-l_{B}^{2} / 2 l_{c}^{2}$ for $l_{c} \gg l_{B}$, with $l_{c}=\sqrt{\hbar / e B_{0}}$ the magnetic length. Fixing $\omega_{-}^{A}=\omega_{+}^{B}=\Omega$, only one free parameter $\omega_{c} \ll \Omega$ remains to specify the two spectra.

We calculate the coupled dynamics of electron and photons from the master equation [13-15],

$$
\frac{d}{d t} \rho=-i[H, \rho]+\mathcal{L}[\rho] .
$$

The commutator describes the coherent evolution of the density matrix $\rho$ under the action of the Hamiltonian $H$ of the dot-cavity system. The operator $\mathcal{L}[\rho]$ describes the coupling of $\operatorname{dot} R$ to the electron reservoir on the right. As we are interested in the entanglement produced by the passage of a single electron through the device, we initialize the system with an electron in $\operatorname{dot} L$. The left reservoir serves only to populate this level initially and is then decoupled, while the coupling to the right reservoir is permanent and acts as a sink for the electron.

The Hamiltonian is $H=\sum_{Y} H_{Y}+H_{T}+H_{+}+H_{-}+$ $H_{\mu}$, with the sum $Y$ taken over all four dots. The Hamiltonians of the dots $A$ and $B$ contain six levels each, according to Eq. (1). For dots $L$ and $R$, we have $H_{L}=$ $E_{L}|L\rangle\langle L|$ and $H_{R}=E_{R}|R\rangle\langle R|$. We set $E_{L}=E_{1}$ and $E_{R}=$ $E_{3}$. The dots are connected via the tunneling Hamiltonian

$$
\begin{aligned}
H_{T}= & \sum_{i=1}^{6}\left\{T_{L A_{i}}|L\rangle\left\langle A_{i}\left|+T_{R A_{i}}\right| R\right\rangle\left\langle A_{i}\left|+T_{L B_{i}}\right| L\right\rangle\left\langle B_{i}\right|\right. \\
& \left.+T_{R B_{i}}|R\rangle\left\langle B_{i}\right|\right\}+ \text { H.c., }
\end{aligned}
$$

where $T_{X Y_{i}}$ are tunnel amplitudes.

The microwave photons have the Hamiltonians $H_{+}=$ $\omega_{-}^{A} b_{+}^{\dagger} b_{+}$and $H_{-}=\omega_{+}^{B} b_{-}^{\dagger} b_{-}$, with $b_{ \pm}$the field operators of $\mathrm{CP}^{ \pm}$microwaves. Since we are on resonance, $\omega_{-}^{A}=$ $\omega_{+}^{B}=\Omega$. In the rotating wave approximation, the emission of microwaves is governed by the Hamiltonian 


$$
\begin{aligned}
H_{\mu}= & g^{A}\left\{\left|A_{3}\right\rangle\left\langle A_{5}|+| A_{2}\right\rangle\left\langle A_{4}|+| A_{1}\right\rangle\left\langle A_{2}\right|\right\} b_{+}^{\dagger} \\
& +g^{B}\left\{\left|B_{3}\right\rangle\left\langle B_{6}|+| B_{2}\right\rangle\left\langle B_{5}|+| B_{1}\right\rangle\left\langle B_{3}\right|\right\} b_{-}^{\dagger}+\text { H.c., }
\end{aligned}
$$

neglecting off-resonant transitions.

The coupling of the right electron reservoir to dot $R$ is incorporated into the master equation through the Lindblad operator $\mathcal{L}[\rho]=D \rho D^{\dagger}-\frac{1}{2} D^{\dagger} D \rho-\frac{1}{2} \rho D^{\dagger} D$. The jump operator is $D=\sqrt{\Gamma_{R}}\left|0_{\text {dot }}\right\rangle\langle R|$, with $\Gamma_{R}$ the tunneling rate and $\left|0_{\text {dot }}\right\rangle$ denoting all four dots empty. In the following, we will for simplicity set $g^{A}=g^{B}=g$ and $T_{X A_{i}}=T_{A}$, $T_{X B_{i}}=T_{B}$ for $X=L, R$ and $i=1, \ldots, 6$. Results of a numerical integration of the master equation (2) are plotted in Figs. 3 and 4.

The density matrix of the field is obtained by tracing out the dot degrees of freedom from the density matrix $\rho(t)$. We denote its (unnormalized) two-photon projection as $\chi(t)$. It has the form

$$
\begin{aligned}
\chi= & r_{+}|++\rangle\left\langle++\left|+r_{-}\right|--\right\rangle\langle--| \\
& +r_{c}|++\rangle\left\langle--\left|+r_{c}^{*}\right|--\right\rangle\langle++| .
\end{aligned}
$$

The mean number of photon pairs in the cavity is given by $N_{2}=\operatorname{Tr} \chi=r_{+}+r_{-}$. This is plotted as a function of time in Fig. 3. The time scale on which the electron is transmitted sequentially through the elements of the system is $\tau=\Gamma_{R}^{-1}+2 g^{-1}+T_{A}^{-1}+T_{B}^{-1}$. We see that for times $t \gg$ $\tau \approx 70 \Omega^{-1}$ the number of photon pairs $N_{2}$ approaches a stationary value, $N_{2}^{\infty}$.

The degree of entanglement (concurrence) $C$ of the photon pair can be calculated using Wootter's formula [16] for the concurrence of the density matrix $\chi(t)$, which in general describes a mixed state. We find

$$
C=\frac{2\left|r_{c}\right|}{r_{+}+r_{-}} \text {. }
$$

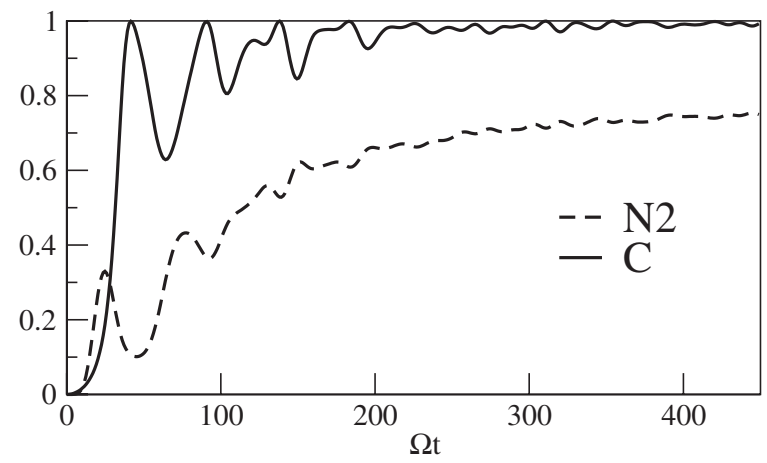

FIG. 3. Dynamics of the microwave entangler. Plotted is the time dependence of the mean number of photon pairs in the cavity $N_{2}$ and their degree of entanglement $C$ (the concurrence). For long times, the field approaches a steady state with, for the weak coupling $T_{A}=T_{B}=0.05 \Omega$ shown here, a high proportion of photon pairs $N_{2}^{\infty} \approx 0.7$ and almost maximal concurrence $C^{\infty} \approx 1$. Model parameters were $\omega_{c}=g=\Gamma_{R}=0.1 \Omega$.
The time-dependent concurrence $C(t)$ is shown in Fig. 3, and this is seen to saturate for $t \gg \tau$. In Fig. 4 (upper panel) we plot this long-time limit $C^{\infty}$ as a function of the coupling asymmetry $T_{A} / T_{B}$ for several values of $T_{A}$.

An analytical solution is possible for $T_{A}, T_{B} \ll \omega_{c}$. Since the top three levels in dots $A$ and $B$ are each separated by $\omega_{c}$, when $T_{A}, T_{B} \ll \omega_{c}$ the electron tunnels from dot $L$ only into the resonant levels at $E_{1}$, producing a photon-pair before leaving the dots. In this limit, the concurrence $C^{\infty}$ of the final state is easily calculated, as the relative amplitudes for the generation of each of the twophoton pairs are proportional to the product of the individual coupling amplitudes along each of the two paths. We find

$$
C=2 \frac{\left|T_{A} T_{B} g_{A} g_{B}\right|^{2}}{\left|T_{A} g_{A}\right|^{4}+\left|T_{B} g_{B}\right|^{4}}
$$

The numerical weak-coupling results in Fig. 4 (crosses) are very close to this analytic result (solid curve). For weak, symmetric interdot couplings, the concurrence approaches unity, corresponding to the production of the Bell state $2^{-1 / 2}(|++\rangle+|--\rangle)$. The negative effects of level
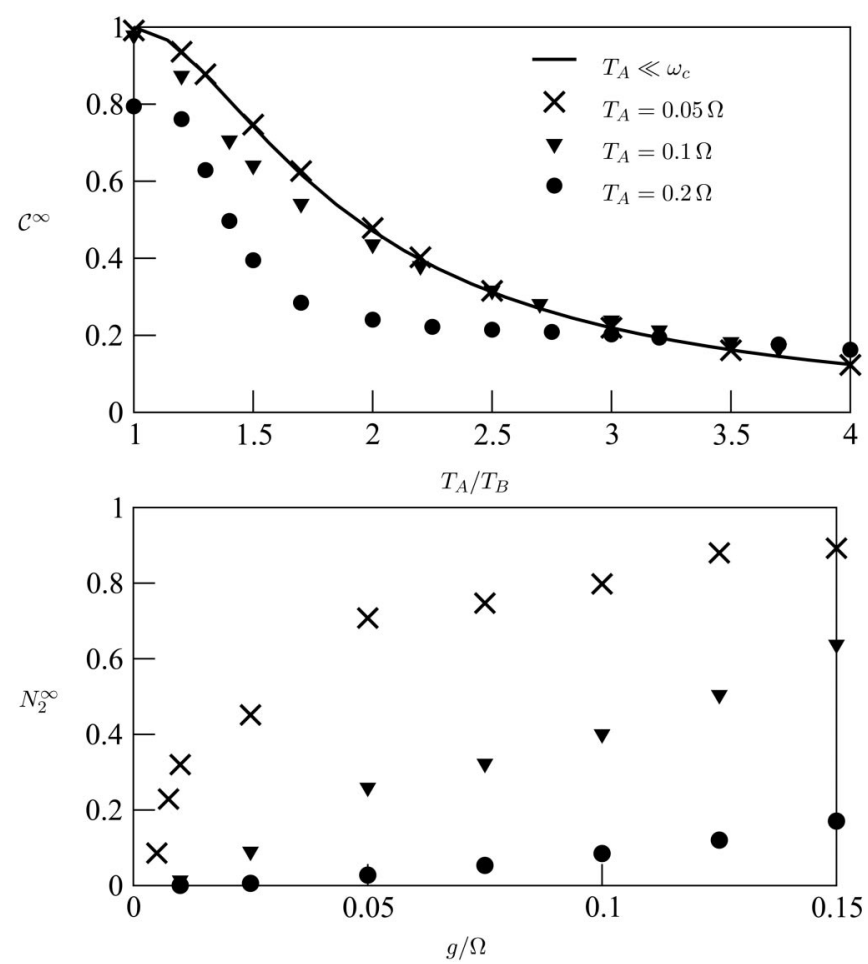

FIG. 4. Performance of the microwave entangler. Upper panel: The asymptotic entanglement of the photon pairs as a function of the asymmetry in the couplings of dots $A$ and $B$ to the other two dots. The solid curve is the analytic result (7) for $T_{A}, T_{B} \ll \omega_{c}$. We fixed $g=\omega_{c}$. Lower panel: Probability $N_{2}^{\infty}$ of successful operation of the entangler as a function of the dot-microwave coupling $g$. The symbols correspond to the same values of $T_{A}$ as in the upper panel, and we fixed $T_{A}=T_{B}$. In both plots, we took $\omega_{c}=\Gamma_{R}=0.1 \Omega$. 


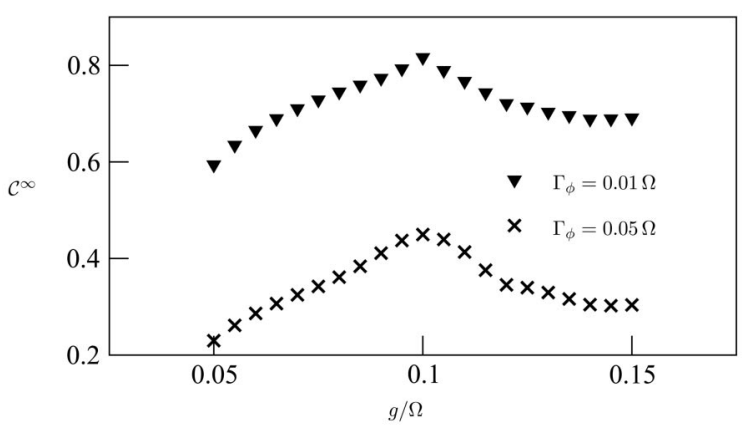

FIG. 5. Effect of decoherence, with rate $\Gamma_{\phi}$, on the asymptotic entanglement of the photon pairs. We fixed $T_{A}=T_{B}=0.05 \Omega$, $\omega_{c}=\Gamma_{R}=0.1 \Omega$, and varied $g$.

broadening induced by the interdot couplings are slight provided $T_{A}, T_{B} \lesssim \omega_{c}$.

Successful operation of the device requires that, after the passage of the electron, the cavity is left with a pair of microwave photons. The probability of successful operation is thus given by the asymptotic mean number of photon pairs $N_{2}^{\infty}$. This probability is plotted in Fig. 4 (lower panel). The success probability tends to unity in the weakcoupling limit.

In the final part of this Letter, we consider two different mechanisms by which the efficiency of the device is reduced. The first mechanism is direct inelastic transitions from upper levels in dots $A$ and $B$ into dot $R$. This reduces the number of photon pairs produced, but provided that the rate of these inelastic processes is smaller than the dotcavity coupling, photon-pair production will still dominate. Moreover, provided that these inelastic processes give no which-way information on the path of the electron, affecting the two dots roughly equally, they have little effect on the degree of entanglement of the photon pairs that are emitted.

The second mechanism is decoherence of the two spatially separated decay paths. The decoherence typically results when the charge on one of the dots couples to other charges in the environment, thereby providing which-way information. We model this charge noise by adding to the master equation (2) jump operators

$$
D_{Y}=\sqrt{\Gamma_{\phi}}\left(\sum_{i}\left|Y_{i}\right\rangle\left\langle Y_{i}\right|\right) \otimes \mathbb{1}_{\text {photon }}
$$

which measure the charge on each of the four dots $Y=A, B, L, R$. Here the sum over $i$ ranges over all states $\left|Y_{i}\right\rangle$ in quantum dot $Y$, the symbol $\mathbb{1}_{\text {photon }}$ denotes the identity for the photon degrees of freedom, and $\Gamma_{\phi}$ is the decoherence rate. The resulting degradation of the concurrence is plotted in Fig. 5, for two values of $\Gamma_{\phi}$. More extensive data indicate that the degradation is algebraic, $C \propto\left(\tau^{*} \Gamma_{\phi}\right)^{-1}$, with $\tau^{*}$ a characteristic time scale of the device that takes on it smallest value when all transition rates $g, T_{A}, T_{B}, \Gamma_{R}$ are close to each other. In Fig. 5 we vary the electron-photon coupling constant $g$, and indeed observe that the concurrence is maximized when $g$ is neither much smaller nor much larger than the other rates. Because the degradation of the concurrence is only algebraic, rather than exponential, a reasonable amount of charge noise can be tolerated.

In summary, we have described a mechanism for the production of entangled microwave photon pairs using intraband transitions in quantum dots. Our calculations indicate that a four-dot device is capable of the output of highly entangled pairs with useful success probability. The entangler may be thought of as an electron interferometer in which each of the two paths is coupled to a different photon-pair producing process. Apart from the different frequency range, it differs from the celebrated biexciton entangler [3] in that the interfering paths are in real space, rather than in energy space, and also in that the correlated polarizations result from orbital selection rules-rather than from spin-orbit coupling.

This work was supported by the Dutch Science Foundation NWO/FOM.

[1] L. Mandel and E. Wolf, Optical Coherence and Quantum Optics (Cambridge University Press, Cambridge, England, 1995).

[2] V. Cerletti, O. Gywat, and D. Loss, cond-mat/0411235; M. Titov, B. Trauzettel, B. Michaelis, and C. W. J. Beenakker, cond-mat/0503676.

[3] O. Benson, C. Santori, M. Pelton, and Y. Yamamoto, Phys. Rev. Lett. 84, 2513 (2000).

[4] O. Gywat, G. Burkard, and D. Loss, Phys. Rev. B 65, 205329 (2002).

[5] T. M. Stace, G. J. Milburn, and C.H.W. Barnes, Phys. Rev. B 67, 085317 (2003).

[6] J. I. Perea and C. Tejedor, cond-mat/0409745.

[7] W. G. van der Wiel, S. de Franceschi, J. M. Elzerman, T. Fujisawa, S. Tarucha, and L. P. Kouwenhoven, Rev. Mod. Phys. 75, 1 (2003).

[8] A. Blais, R.-S. Huang, A. Wallraff, S. M. Girvin, and R. J. Schoelkopf, Phys. Rev. A 69, 062320 (2004).

[9] L. Childress, A.S. Sørensen, and M.D. Lukin, Phys. Rev. A 69, 042302 (2004).

[10] J. Gabelli, L.-H. Reydellet, G. Fève, J.-M. Berroir, B. Plaçais, P. Roche, and D. C. Glattli, Phys. Rev. Lett. 93, 056801 (2004).

[11] L. P. Kouwenhoven, D. G. Austing, and S. Tarucha, Rep. Prog. Phys. 64, 701 (2001).

[12] The quantum numbers $n_{ \pm}^{Y}$ are related to the set $\{n, l\}$ of Ref. [11] via $n=\min \left(n_{+}, n_{-}\right)$and $l=n_{-}-n_{+}$.

[13] T. H. Stoof and Yu. V. Nazarov, Phys. Rev. B 53, 1050 (1996).

[14] S. A. Gurvitz, Phys. Rev. B 57, 6602 (1998).

[15] T. Brandes, Phys. Rep. 408, 315 (2005).

[16] W. K. Wootters, Phys. Rev. Lett. 80, 2245 (1998). 